\section{Wide-range continuously tunable microwave photonic filter using high- birefringence linearly chirped fiber Bragg grating and polarization beamsplitters}

\author{
J. Q. Zhou, Member SPIE, S. Aditya, \\ P. Shum, MEMBER SPIE, L. Xia, and B. P. Parhusip \\ Nanyang Technological University, Network Technology \\ Research Centre, Research TechnoPlaza, \\ 4th Storey, XFrontier Block, 50 Nanyang Drive, \\ Singapore 637553 \\ E-mail: jqzhou@pmail.ntu.edu.sg
}

\begin{abstract}
A continuously tunable, coherence-free microwave photonic notch filter is proposed and demonstrated experimentally. This filter is based on two polarization beamsplitters with a high-birefringence linearly chirped fiber Bragg grating used as the tunable component. High stability is obtained. The polarization-maintaining structure is free from the random optical interference problem. By adjusting the operating wavelength, more than $5 \mathrm{GHz}$ free-spectral-range tunability with $40-\mathrm{dB}$ notch rejection is achieved. ( 2009 Society of Photo-Optical Instrumentation Engineers.

[DOI: 10.1117/1.3059623]
\end{abstract}

Subject terms: microwave photonic filter; notch filter; continuously tunable; linearly chirped fiber Bragg grating; polarization beamsplitter; coherence free.

Paper 080774LR received Sep. 30, 2008; revised manuscript received Oct. 29, 2008; accepted for publication Nov. 17, 2008; published online Jan. 7, 2009.

\section{Introduction}

Processing microwave and millimeter wave signals in the optical domain using microwave photonic filters (MPFs) has attracted considerable interest due to the advantages of high time-bandwidth product, immunity to electromagnetic interference (EMI), tunability, and low loss. ${ }^{1,2}$ To achieve microwave signal processing by optical means, various schemes of optical signal division, delay, and summing have been proposed. Incoherent summing of optical signals is more attractive in practice than a coherent approach because the former is insensitive to polarization fluctuation caused by environmental perturbation. To satisfy the requirement of incoherent summing, either the laser coherence time must be shorter than the optical delay time, or the state of polarization (SOP) of the optical signals must be orthogonal. For the first approach, a laser array ${ }^{3}$ or a sliced wideband source ${ }^{4}$ is used, but these increase the complexity of the system. If a single-wavelength laser source is used, either a long time delay line is required ${ }^{5}$ which restricts the free spectral range (FSR), or the optical mixing effect is used ${ }^{6}$ which increases the system cost. The second ap-

0091-3286/2009/\$25.00 @ 2009 SPIE

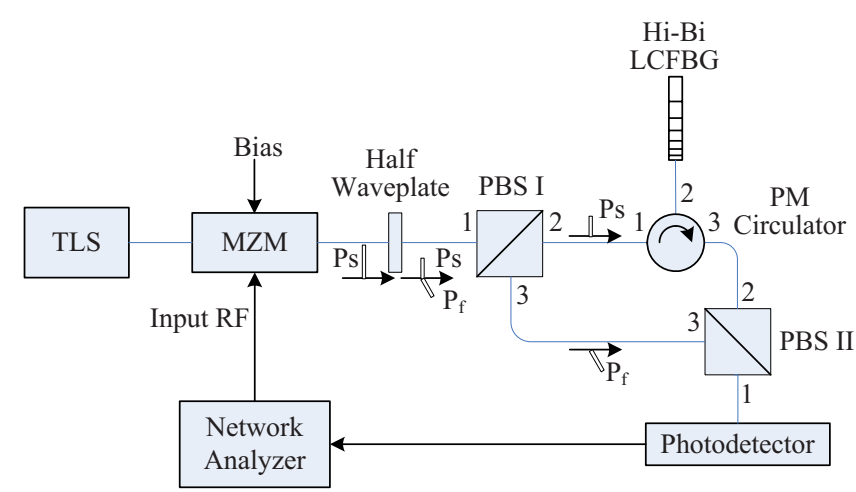

Fig. 1 Schematic of the experimental setup: TLS; tunable laser source; MZM, Mach-Zehnder modulator; PBS, polarization beamsplitter; LCFBG, linearly chirped fiber Bragg grating.

proach was demonstrated in Ref. 7; however, it has only step tuning ability. Continuous tunability was achieved in an MPF that consisted of a high-birefringence linearly chirped fiber Bragg grating (Hi-Bi LCFBG), a polarization beamsplitter (PBS), and a Hi-Bi coupler. ${ }^{8}$ However, in Ref. 8 the FSR tuning range induced by applying uniform strength to the grating is relative by small. Also, the notch rejection may be affected for this configuration, with a LCFBG inside a fiber loop, because the limited reflectivity of the grating causes signal crosstalk. Further, the Hi-Bi fiber coupler also induces 6-dB power losses.

In this paper, we propose a continuously tunable MPF that uses a Hi-Bi LCFBG as the variable time delay element. Two PBSs are used to split and combine the optical signals; this avoids the power loss of a Hi-Bi coupler and increases the stability dramatically. By using a polarizationmaintaining structure, the filter is free from the problem of random optical interference. The FSR tunability is achieved by adjusting the optical path length of the upper arm through changing the operating wavelength; this avoids mechanical movement. The time delay difference induced by this approach is much larger compared with Ref. 6. FSR tunability of $5 \mathrm{GHz}$ and $40 \mathrm{~dB}$ of notch rejection are obtained.

\section{Filter Configuration and Principle of Operation}

Figure 1 shows the experimental setup for the proposed MPF. A continuous lightwave from a tunable laser source (TLS) is modulated by a Mach-Zehnder modulator (MZM), which is driven by an rf signal from a network analyzer. The output of the MZM is fed to a half waveplate, which is used to excite two orthogonal and equal power linear SOP components along the slow and fast axes of the polarization-maintaining fiber (PMF). These two components are then split by PBS I. The component along the slow axis goes to the upper arm, gets reflected by a $\mathrm{Hi}-\mathrm{Bi}$ LCFBG, and reaches PBS II. The component along the fast axis propagates in the lower arm and goes to PBS II directly.

For a chirped grating, the strongest mode coupling occurs at the Bragg wavelength $\lambda_{B}$, which is the center wavelength of the input light that can be back-reflected from the grating. The Bragg wavelength is a function of grating pitch $\Lambda(z)$ : 


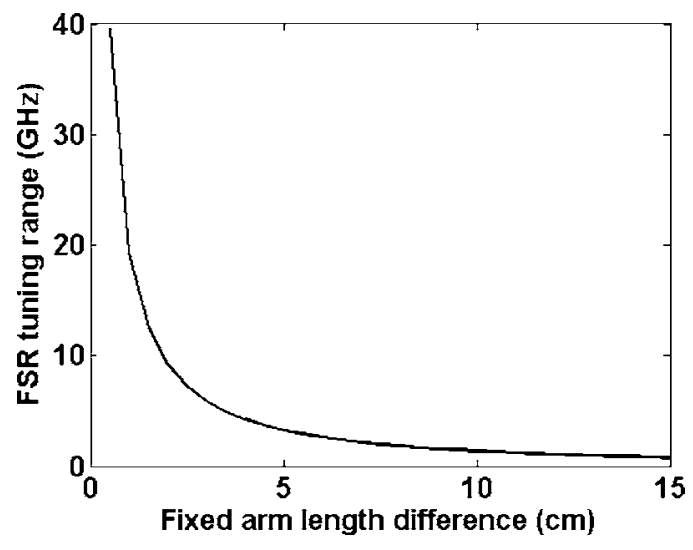

Fig. 2 Relation between fixed difference between arm lengths and FSR tuning range.

$\lambda_{B}=2 n_{\mathrm{eff}} \Lambda(z)$,

where $n_{\text {eff }}$ is the effective refractive index of the fiber. A linearly chirped grating has a variable pitch that is a linear function of distance along the grating so that the Bragg wavelength and reflection location along the grating forms a linear relation. A Hi-Bi LCFBG acts like two gratings corresponding to the two orthogonal polarization axes with the same pitch function. We use only the LCFBG along the slow axis. The reason for using the $\mathrm{Hi}-\mathrm{Bi}$ grating here is to maintain the linear SOP. When the two components reach PBS II, they are recombined and output from port 1. Since the connection of PBS II is just the reverse of that of PBS I, the two components at PBS II port 1 are orthogonal. Thus, incoherent summing is achieved at the photodetector and no power loss occurs during the combination.

The MPF is a two-tap transversal filter, and its normalized transfer function is

$|H(f)|=|\cos (\pi f \Delta T)|$,

where $f$ is the rf frequency, and $\Delta T$ is the total time delay difference between the two arms.

Let the fiber lengths of the upper and lower arms be $L_{1}$ (without including the length of the Hi-Bi LCFBG) and $L_{2}$, and let the distance from the grating input to the reflection point be $L_{g}$. We can express $\Delta T$ as

$\Delta T=\frac{\left(L_{1}+2 L_{g}\right) n_{s}-L_{2} n_{f}}{c}$,

where $n_{s}$ and $n_{f}$ are the effective refractive indices of the slow and fast axes of the PMF. Since $n_{s} \approx n_{f} \equiv n_{g}$, Eq. (3) becomes

$\Delta T \approx \Delta L n_{g} / c+2 L_{g} n_{g} / c=\tau_{0}+\tau_{g}$,

where $\Delta L=L_{1}-L_{2}$ is the fixed difference between arm lengths; $\tau_{0}=\Delta L n_{g} / c$ and $\tau_{g}=2 L_{g} n_{g} / c$ are the fixed and tunable time delay differences, respectively; and $n_{g}$ is the average refractive index. In Eqs. (3) and (4), the time delay difference caused by the birefringence of the fiber pigtails of port 1 of the two PBSs is omitted because the pigtail length is only tens of centimeters. The FSR of the filter is described by

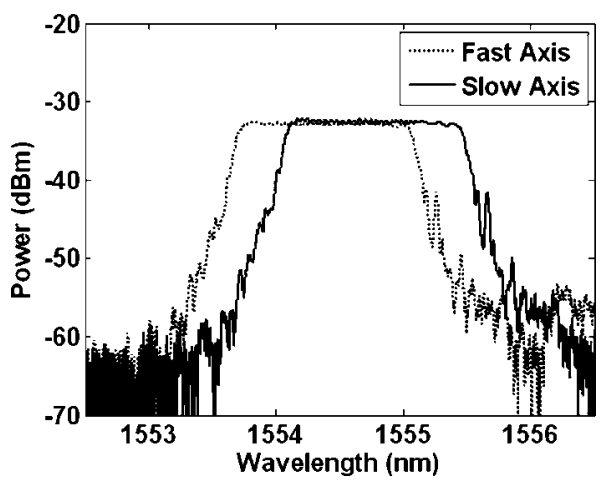

Fig. $3 \mathrm{Hi}-\mathrm{Bi}$ LCFBG reflection spectra.

$\mathrm{FSR}=\frac{1}{\Delta T}=\frac{1}{\tau_{0}+\tau_{g}}$.

From Eqs. (4) and (5), we can see that the FSR can be tuned by adjusting $\tau_{g}$ through changing the operating wavelength because $L_{g}$ is a linear function of wavelength. The FSR tuning range also depends on the fixed arm length difference $\Delta L$ or $\tau_{0}$. Figure 2 shows the calculated relation between $\Delta L$ and FSR tuning range when $L_{g}$ is $10 \mathrm{~cm}$.

\section{Experimental Results and Discussion}

The Hi-Bi LCFBG used in the experiments was fabricated by exposing a hydrogen-loaded PMF to a 244-nm UV laser beam through a linearly chirped phase mask with a length of $10 \mathrm{~cm}$. Figure 3 shows the measured reflection spectra for the fast and slow axes, respectively. In our experiments, the slow axis of the Hi-Bi LCFBG is used with an operating wavelength ranging from 1554.1 to $1555.2 \mathrm{~nm}$. The grating short-wavelength port is connected with PM circulator port 2, so the shorter operating wavelength corresponds to a higher FSR.

The measured frequency response of the proposed filter (shown in Fig. 4) agrees well with the theoretical calculation. When the fixed arm length difference $\Delta L$ is $3.1 \mathrm{~cm}$, the measured FSR at operating wavelengths 1554.1, $1554.5,1555$, and $1555.2 \mathrm{~nm}$ are $6.65,2.88,1.67$, and $1.48 \mathrm{GHz}$, respectively. More than a 5-GHz FSR tuning range is achieved. As indicated by Fig. 2, the FSR tuning

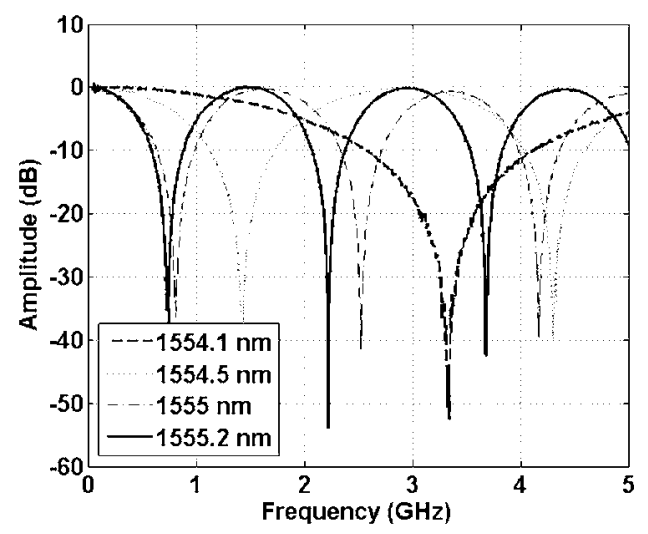

Fig. 4 Measured frequency response when the fixed arm length difference is $3.1 \mathrm{~cm}$. 


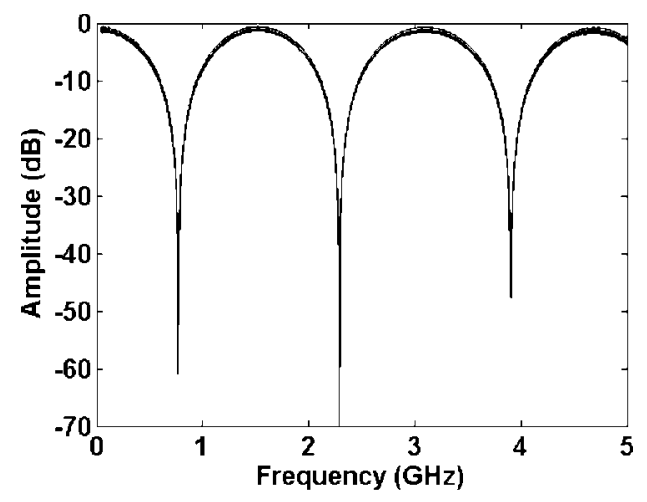

(a)

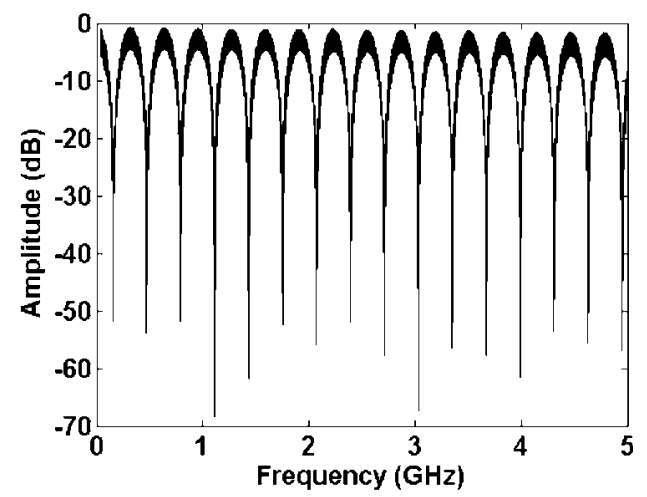

(b)

Fig. 5 Stability measurement over $1 \mathrm{~h}$ with 10-s intervals for (a) the proposed filter structure and (b) the structure using a PM coupler to replace PBS II.

range is also determined by $\Delta L$. If we add a polarizationmaintaining time delay line in one of the arms for adjusting $\tau_{0}$, FSR tunability can be extended further.

The frequency response of the proposed filter is stable because the polarization-maintaining structure greatly reduces the SOP fluctuation caused by environmental perturbation. Another major reason is the application of PBSs. A PBS has a high extinction ratio between ports 2 and 3, e.g., $30 \mathrm{~dB}$ in our case. By using the PBSs as beamsplitting and combining components, the crosstalk between the slow and fast axes at the second PBS port 1 is suppressed. Figure 5 (a) shows the results of measurements for $1 \mathrm{~h}$ with intervals of $10 \mathrm{~s}$ between each measurement when the operating wavelength is $1555.2 \mathrm{~nm}$. Very good stability can be seen. The fluctuation of the peaks is less than $1 \mathrm{~dB}$ and the notches are all greater than $40 \mathrm{~dB}$. For comparison, similar measurements were carried out with PBS II replaced by a PM coupler (extinction ratio $21 \mathrm{~dB}$ ). In this case, Fig. 5(b) shows that the peak variation is about $3.5 \mathrm{~dB}$ and notches range from 25 to $50 \mathrm{~dB}$. The small FSR in Fig. 5(b) is due to the pigtails of PM coupler, which increase the fixed arm length difference.

\section{Conclution}

In this letter, we demonstrated a continuously tunable microwave photonic notch filter that is based on two PBSs and a Hi-Bi linearly chirped fiber Bragg grating as tuning element. The PM structure is free from the problem of random optical interference and has very stable frequency response. A 1-h measurement with intervals of $10 \mathrm{~s}$ shows only $1 \mathrm{~dB}$ of fluctuation. Experimental results demonstrate wide-range FSR tunability with very deep notches in excess of $40 \mathrm{~dB}$.

\section{Acknowledgments}

This work is partially supported by a grant from the Agency for Science, Technology and Research, Singapore.

\section{References}

1. J. Capmany, B. Ortega, D. Pastor, and S. Sales, "Discrete-time optical processing of microwave signals," J. Lightwave Technol. 23, 702723 (2005).

2. R. A. Minasian, "Photonic signal processing of microwave signals," IEEE Trans. Microwave Theory Tech. 54, 832-846 (2006).

3. V. Polo, B. Vidal, J. L. Corral, and J. Marti, "Novel tunable photonic microwave filter based on laser arrays and $\mathrm{N} \times \mathrm{N}$ AWG-based delay lines," IEEE Photon. Technol. Lett. 15, 584-586 (2003).

4. J. Mora, B. Ortega, A. Diez, J. L. Cruz, M. V. Andres, J. Capmany, and D. Pastor, "Photonic microwave tunable single-bandpass filter based on a Mach-Zehnder interferometer," J. Lightwave Technol. 24, 2500-2509 (2006).

5. W. Zhang, J. A. R. Williams, L. A. Everall, and I. Bennion, "Fibre optic radio frequency notch filter with linear and continuous tuning by using a chirped fibre grating," Electron. Lett. 34, 1770-1772 (1998).

6. J. Wang and J. Yao, "A tunable photonic microwave notch filter based on all-optical mixing," IEEE Photon. Technol. Lett. 18, 382-384 (2006).

7. W. Zhang, J. A. R. Williams, and I. Bennion, "Polarization synthesized optical transversal filter employing high birefringence fiber gratings," IEEE Photon. Technol. Lett. 13, 523-525 (2001).

8. X. K. Yi, C. Lu, X. F. Yang, W. D. Zhong, F. Wei, L. Ding, and Y. X. Wang, "Continuously tunable microwave-photonic filter design using high-birefringence linear chirped grating," IEEE Photon. Technol. Lett. 15, 754-756 (2003). 